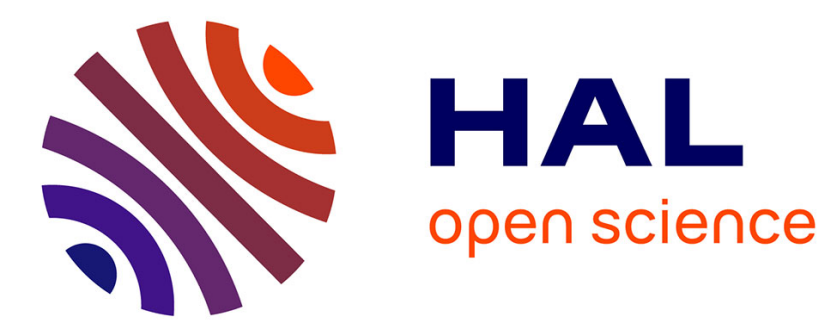

\title{
Les directeurs commerciaux face au processus budgétaire
}

\author{
Madeleine Besson, Hélène Löning, Carla Mendoza
}

\section{To cite this version:}

Madeleine Besson, Hélène Löning, Carla Mendoza. Les directeurs commerciaux face au processus budgétaire. 25ème congrès de l'Association francophone de comptabilité (AFC). "Normes et Mondialisation", Association francophone de comptabilité (AFC), May 2004, Orléans, France. pp.CD-Rom. halshs-00592971

\section{HAL Id: halshs-00592971 https://shs.hal.science/halshs-00592971}

Submitted on 13 May 2011

HAL is a multi-disciplinary open access archive for the deposit and dissemination of scientific research documents, whether they are published or not. The documents may come from teaching and research institutions in France or abroad, or from public or private research centers.
L'archive ouverte pluridisciplinaire HAL, est destinée au dépôt et à la diffusion de documents scientifiques de niveau recherche, publiés ou non, émanant des établissements d'enseignement et de recherche français ou étrangers, des laboratoires publics ou privés. 


\section{LES DIRECTEURS COMMERCIAUX FACE AU PROCESSUS BUDGETAIRE}

Madeleine Besson, Professeur associé, INT- département Sciences de Gestion

9, rue Charles Fourier 91011 Evry Cedex France, téléphone : +33 1607641 30, courrier électronique : madeleine.besson@int-evry.fr

Hélène Löning, Professeur associé, HEC - département Comptabilité-Contrôle de gestion

1, rue de la Libération, 78350 Jouy-en- Josas, téléphone : +33 1396772 63, courrier électronique : loning@hec.fr

Carla Mendoza, Professeur associé, ESCP-EAP - département CPO -

79, avenue de la République - 75543 Paris cedex 11 - France, téléphone : +33.1.49.23.20.52, courrier électronique : mendoza@escp-eap.net

Adresse de correspondance : Carla Mendoza.

\section{Résumé}

Cette communication explore le concept de pression budgétaire, désigné en anglais par Budget Emphasis ou Reliance on Accounting Performance Measures $(R A P M)$ à partir d'une approche exploratoire fondée sur des entretiens en profondeur auprès de 14 directeurs commerciaux. Elle analyse également les inter relations entre la pression budgétaire et la participation budgétaire et examine les effets de la pression budgétaire sur les comportements.

Mots clés: pression budgétaire, évaluation, participation budgétaire, slack, directeurs

\begin{abstract}
This study relies on an exploratory approach based on in-depth interviews with 14 commercial directors, in order to explore the dimensions of budget emphasis, also referred to as Reliance on Accounting Performance Measures (RAPM). It analyses the relationships between budget emphasis and budget participation and examines the consequences of budget emphasis on managers' behaviour.
\end{abstract}

Key words: budget emphasis, Reliance on Accounting Performance Measures, evaluation, budget participation, slack, sales and marketing managers 


\section{Introduction}

La pression budgétaire, ses causes et ses conséquences sur les comportements ont alimenté un important courant de recherche depuis plusieurs décennies dans le monde anglo-saxon. Le concept de pression budgétaire, désigné en anglais par Budget emphasis ou, dans les études plus récentes par Reliance on Accounting Performance Measures, désigne des situations où les managers se voient soumis à une forte pression pour atteindre leurs objectifs budgétaires : en particulier, l'évaluation de leur performance dépend très largement du respect de ceux-ci. Ainsi que l'attestent plusieurs revues de la littérature sur le sujet (Briers et Hirst, 1990 ; Naro, 1998 ; Hartmann, 2000; Langevin et Naro, 2003), ces travaux représentent une masse conséquente, avec de nombreuses tentatives d'intégration ou de réplication de recherches empiriques. Malgré une sophistication croissante des outils d'analyse statistique utilisés, ces différents travaux ont produit des résultats partiellement contradictoires et n'ont pas permis d'aboutir à des conclusions claires. L'une des principales raisons avancées pour expliquer cette situation est l'insuffisante spécification des concepts utilisés et en tout premier lieu de la variable centrale qu'est la pression budgétaire. C'est pourquoi, les objectifs de notre recherche sont d'explorer les dimensions de la pression budgétaire et ses effets sur les comportements. Nous avons choisi de nous focaliser sur une fonction particulière: les directions commerciales. En effet, ces managers ont généralement des objectifs budgétaires précis (budget de ventes, budget de frais commerciaux). Il est fréquent qu'une forte pression soit placée sur l'atteinte des objectifs commerciaux dans la mesure où les résultats de l'entreprise sont souvent largement tributaires de ces derniers. Par conséquent, les directions commerciales nous ont semblé constituer un terrain privilégié pour observer les phénomènes de pression budgétaire à l'œuvre. Ce qui nous importait, c'était de comprendre comment les managers percevaient la pression budgétaire et comment ils y faisaient face. C'est pourquoi, nous avons mobilisé une approche exploratoire fondée sur des entretiens en profondeur et le recours à des méthodes projectives. Les résultats obtenus mettent en lumière des situations plus complexes que celles décrites par les travaux du courant RAPM. Par exemple, il est apparu que la pression budgétaire constitue un processus continu prenant place tout au long de l'année et non uniquement au moment de l'évaluation des performances. Par ailleurs, la pression budgétaire ne s'exerce pas seulement au niveau des individus mais est gérée de manière plus collective. Plus généralement, nos résultats apportent un éclairage renouvelé sur le concept de pression budgétaire et sur les comportements développés par les managers face à celle-ci.

Nous commencerons par examiner la littérature consacrée à la pression budgétaire et par préciser les objectifs de notre étude. Nous présenterons ensuite la méthodologie de recherche retenue, les résultats et nos conclusions.

\section{Analyse de la littérature et objectifs de la recherche}

Le budget constitue une composante essentielle du système de contrôle. Il peut jouer différents rôles: instrument de coordination et de communication, outil de gestion prévisionnelle et dispositif de délégation et de motivation (Bouquin, 2001). 
Les processus budgétaires (élaboration des budgets et contrôle budgétaire) ont généralement été présentés comme une démarche volontariste au travers de laquelle l'entreprise signifie à tous ses responsables qu'il ne s'agit pas de prévoir de manière passive mais de se fixer des objectifs (Dupuy, Rolland, 1991). Les budgets doivent donc traduire l'engagement de chaque responsable devant la direction de l'entreprise (Lauzel et Teller, 1997). En ce sens, le budget peut être considéré comme un contrat passé entre un responsable et la hiérarchie portant sur des obligations de moyens et/ou de résultats (Bouquin, 2001). Cette logique contractuelle a notamment marqué l'introduction du budget dans les firmes américaines. Dans celles-ci, le budget représente un contrat au travers duquel les responsables de divisions ont l'obligation d'atteindre les résultats budgétés en contrepartie du pouvoir sur les ressources mises à leur disposition (Escoffier, 1999). Ainsi, les budgets ont rapidement été associés à une Direction Par Objectifs (DPO) et à un système de sanctions/récompenses ce qui peut être une source de motivation ou de mécontentement (Gervais, 1999, 2000).

Aux Etats-Unis, les premiers travaux sur les aspects humains et comportementaux de la gestion budgétaire ont cherché à montrer que la manière dont les budgets sont utilisés peut générer des tensions et des dysfonctionnements (Argyris, 1952).

Par la suite, l'étude de Hopwood (1972) a constitué le point de départ d'un programme de recherche dans les pays anglo-saxons. Cette étude a mis en évidence l'existence de différents styles de gestion budgétaire dont un dans lequel l'évaluation des performances est étroitement fondée sur la capacité du responsable à respecter strictement ses objectifs budgétaires à court terme. Ce travail de recherche fondateur a donné naissance au concept de pression budgétaire. Les déterminants et les conséquences de la pression budgétaire ont été largement explorés par la suite (Otley, 1978 ; Kenis, 1979 ; Brownell, 1982, 1985 ; Hirst, 1983, 1987 ; Govindarajan, 1984 ; Brownell et Hirst, 1986 ; Imoisili, 1989 ; Briers et Hirst, 1990 ; Brownell et Dunk, 1991 ; Harrison, 1992 ; Dunk, 1989, 1992, 1993 ; Shields et Young, 1993 ; Shields et Shields, 1998).

Dans un premier temps, nous examinons les études consacrées à la pression budgétaire et à ses conséquences. Ensuite, nous passons en revue quelques questionnements récents à l'égard de cet ensemble de travaux de recherche.

\subsection{La pression budgétaire et ses conséquences}

Quels sont les effets produits par une évaluation des performances strictement fondée sur la capacité d'un manager à respecter son budget? L'hypothèse initialement testée (Hopwood, 1972 ; Otley, 1978) est qu'un fort degré de pression budgétaire a des conséquences négatives sur les comportements et aboutit à des performances moindres. Hopwood (1972), en particulier, a montré que le respect du budget, comme critère essentiel pour évaluer les performances d'un manager, aboutissait à des comportements dysfonctionnels et générait des tensions dans les relations au travail (Job Related Tensions - JRT). Otley (1978), en tentant de reproduire les résultats de Hopwood (1972) est parvenu à des résultats opposés. Il a observé qu'un fort degré de pression budgétaire était corrélé positivement avec les performances individuelles (Otley, 1978). Un large courant de recherche s'est alors ouvert afin d'identifier les variables de contingence susceptibles d'expliquer ces résultats contradictoires. Une variable a été particulièrement étudiée : il s'agit de la participation budgétaire. 


\subsubsection{Pression budgétaire et participation budgétaire}

La participation budgétaire traduit la possibilité qu'ont les managers de participer à la préparation de leurs budgets et d'avoir une influence sur leurs objectifs budgétaires (Kenis, 1979). De nombreuses recherches ont suggéré que, lorsque les managers peuvent participer à la fixation de leurs objectifs budgétaires, il les acceptent plus volontiers et s'efforcent plus activement de les atteindre (Argyris, 1952 ; Wallace, 1966 ; Hanson, 1966 ; Dunbar, 1971).

Par exemple, Milani (1975) a mis en évidence des relations positives et significatives entre la participation à l'établissement du budget et les attitudes développées vis-à-vis du travail et de l'entreprise. En revanche, ses résultats ont révélé une relation faible entre participation et performance du manager. Hofstede (1967) a noté des attitudes positives chez les subordonnés qui avaient la possibilité d'intervenir dans la fixation des budgets. De manière similaire, Swieringa et Moncur (1975) ont observé un degré de satisfaction plus élevé chez les managers qui étaient consultés lors de l'établissement de leur budget que chez ceux qui ne l'étaient pas. D'autres travaux ont observé l'effet de la participation budgétaire quand elle est couplée à une forte pression budgétaire. Brownell (1982) est ainsi parvenu à démontrer que la participation budgétaire joue le rôle d'une variable modératrice entre la pression budgétaire et les effets que cette dernière produit. Une forte pression mise sur l'atteinte des objectifs budgétaires aurait un effet positif sur la performance du manager lorsque ce dernier a la possibilité de participer activement à l'établissement de ses objectifs budgétaires.

Enfin, les chercheurs du courant RAPM se sont attachés à étudier l'ensemble des relations croisées entre la pression budgétaire, la participation à l'élaboration du budget et l'incertitude (Brownell et Hirst, 1986; Dunk, 1989; Brownell et Dunk, 1991) mais les résultats successivement obtenus sont partiellement contradictoires et ne permettent pas de dégager des conclusions claires.

D'autres travaux du courant RAPM ont plus particulièrement exploré les effets de la pression budgétaire sur les comportements.

\subsubsection{Les conséquences de la pression budgétaire sur les comportements : création de slack, orientation court terme et manipulation des données}

L'hypothèse la plus courante dans la littérature est que la pression budgétaire a des conséquences négatives sur les comportements. En cas de forte pression budgétaire, les managers qui ne parviennent pas à atteindre leurs objectifs se trouvent confrontés à des interventions de leur hiérarchie, à une perte de leurs primes et éventuellement à une perte de leur travail (Merchant et Manzoni, 1989). Une voie possible, pour se protéger contre un tel risque, consiste à créer du slack, c'est-à-dire à biaiser les estimations budgétaires pour rendre les budgets plus facilement atteignables (Onsi, 1973 ; Lukka, 1988 ; Kren, 1993). Cependant, les recherches empiriques sur les relations entre degré de pression budgétaire et création de slack n'ont pas abouti à des résultats convergents. Ainsi, certains travaux ont montré que le slack était faible lorsque la pression budgétaire était forte (Dunk, 1993 ; Merchant, 1985). Une deuxième conséquence d'une forte pression budgétaire est le risque de focalisation excessive sur les résultats à court terme au détriment de la compétitivité à long terme (Hayes et Abernethy, 1980 ; Merchant et Bruns, 1986 ; Merchant, 1990 ; Laverty, 1996 ; Van der Stede, 2000). Enfin, la pression budgétaire aurait comme autre effet négatif de conduire les managers à manipuler les mesures de performance afin d'afficher de bons résultats (Merchant, 1990). 


\subsection{Des questionnements récents}

Alors qu'en 1991, Brownell et Dunk indiquent que "le courant permanent de travaux consacrés à cette question (RAPM) représente la seule masse critique de travail organisée en contrôle de gestion jusqu'à présent » (Brownell et Dunk, 1991, p. 703), on peut s'interroger sur le caractère réellement organisé de cet ensemble (Chapman, 1997 ; Hartmann, 2000 ; Otley et Fakiolas, 2000). D'une part, les hypothèses d'un grand nombre d'études empiriques n'ont pas pu être confirmées. D'autre part, on note peu de convergences dans les résultats produits. Les tentatives pour reproduire les résultats de recherches antérieures ont rarement été couronnées de succès. Par exemple, Brownell et Hirst (1986) et Ross (1995) ne sont pas parvenus à reproduire les résultats de Hirst (1983). De même, Hirst (1987), Dunk (1989) et Aranya (1990) ont tenté sans succès de reproduire ceux de Brownell (1982), etc.

Plusieurs explications ont été avancées pour tenter d'analyser cette situation.

Premièrement, la façon de mesurer les variables a changé d'une étude à l'autre, et on est donc en droit de se demander si les chercheurs ont réellement travaillé sur les mêmes concepts. En particulier, le terme de «pression budgétaire» recouvre une diversité de mesures et de concepts (Otley et Fakiolas, 2000 ; Vagneur et Peiperl, 2000). Certains chercheurs - Otley (1978), Brownell (1982), Brownell et Hirst (1986) - ont utilisé un questionnaire proche de celui élaboré par Hopwood (1972) et ont adopté la même approche pour faire apparaître les différents styles d'évaluation. En particulier, les situations correspondant à une forte pression budgétaire sont celles dans lesquelles le manager a cité « le respect de mon budget» parmi les trois premiers critères d'évaluation de ses performances (technique de ranking). Dans d'autres travaux, la pression budgétaire est mesurée en examinant jusqu'à quel point l'évaluation des performances est fondée sur des données quantitatives (Hirst, 1983 ; Hirst et Yetton, 1984). Enfin, certains chercheurs ont utilisé un autre instrument de mesure qui oppose une évaluation des performances fondée sur des mesures objectives et une évaluation dans laquelle des éléments subjectifs sont pris en compte (Govindarajan, 1984 ; Govindarajan et Gupta, 1985). Toutes ces études ont porté sur la pression budgétaire mais il n'est pas certain qu'elles aient travaillé sur le même concept (Vagneur et Peiperl, 2000).

En second lieu, le courant de recherche RAPM souffrirait aussi d'un développement théorique insuffisant (Hartmann, 2000). Alors qu'un très grand nombre de variables de contingence ont été étudiées et de nombreuses hypothèses testées, la justification théorique des hypothèses émises reste faible (Briers et Hirst, 1990). La plupart des études ne distinguent pas assez clairement les prédictions concernant les effets produits par la pression budgétaire des prédictions ayant trait aux facteurs explicatifs de celle-ci (Hartmann, 2000).

Malgré les critiques examinées ci-dessus, les questions soulevées par les travaux consacrés aux déterminants et aux effets du degré de pression budgétaire demeurent essentielles. Jusqu'où faut-il prendre en compte l'atteinte des objectifs budgétaires dans l'évaluation des managers ? Comment les managers réagissent-ils face à une forte pression budgétaire ?

\subsection{Objectifs de la recherche}

La recherche présentée dans cet article vise à apporter des éléments de réponse à ces questions.

Notre premier objectif est de mieux comprendre la variable « degré de pression budgétaire ». Nous examinons les caractéristiques des situations où le manager a le sentiment de subir une 
forte pression pour respecter ses objectifs budgétaires afin d'analyser les différentes dimensions de la pression budgétaire.

Notre deuxième objectif est d'étudier le degré et les modalités de participation des managers aux processus budgétaires. La plupart des travaux sur la pression budgétaire ont examiné si un manager avait la possibilité d'intervenir auprès de sa hiérarchie (et jusqu'à quel point) au moment de la fixation de ses objectifs budgétaires. Nous examinons la question de la participation au processus budgétaire de manière plus complète, en analysant les interactions entre les différents niveaux hiérarchiques. En effet, un manager a un supérieur mais aussi des collaborateurs et le processus budgétaire se déroule aux différents échelons de la ligne hiérarchique.

Enfin, le troisième objectif de cette étude est de comprendre les effets produits par le degré de pression budgétaire et par le degré de participation au processus budgétaire sur les comportements et attitudes des managers. Ainsi que nous l'avons indiqué plus haut, les résultats produits par le courant RAPM sont partiellement contradictoires. C'est le cas par exemple des relations entre pression budgétaire et création de slack.

En définitive, nous poursuivons deux finalités : $1^{\circ}$ mieux comprendre ce que recouvrent les variables «degré de pression budgétaire » et «participation au processus budgétaire »; $2^{\circ}$ identifier leurs interrelations et leurs effets sur les comportements. Le schéma ci-dessous synthétise les variables examinées dans le cadre de cette recherche.

Figure 1

Cadre conceptuel de la recherche

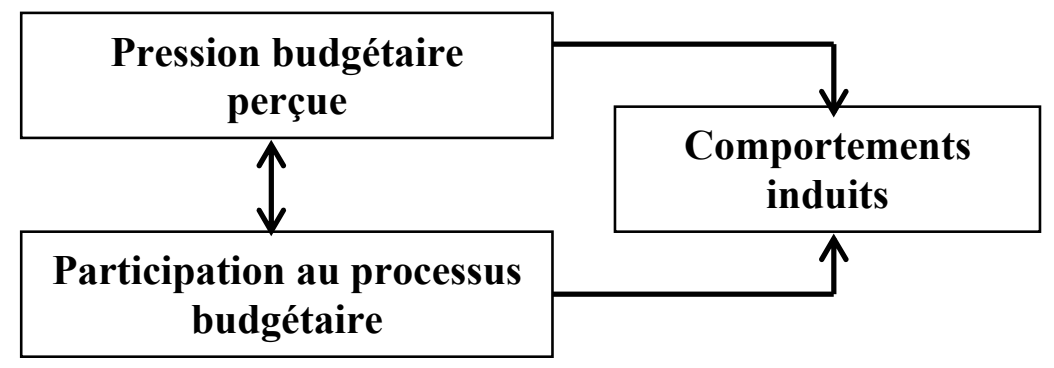

A ce stade, il convient de souligner que nous n'avons pas cherché, dans cet article, à évaluer l'impact de variables modératrices, sans doute importantes, telles que le type de stratégie poursuivie par l'entreprise, le degré d'incertitude de l'environnement ou de la tâche, etc. Mais, il nous semblait nécessaire, dans un premier temps, de mieux comprendre la principale variable du courant RAPM, à savoir la pression budgétaire et d'examiner ses effets sur les comportements des managers.

\section{Méthode de recherche}

Les travaux du courant RAPM ont généralement eu recours à des enquêtes par questionnaires et ont analysé les réponses recueillies avec des méthodes statistiques. Nous avons opté pour une méthodologie de recherche qualitative fondée sur des entretiens semi-directifs d'une heure et demie en moyenne auprès de directeurs commerciaux de grandes sociétés installées en France. Dans les paragraphes qui suivent, nous décrirons les caractéristiques des 
responsables interrogés, le dispositif de recueil des données et les modalités d'analyse des résultats.

\subsection{La cible}

Elle est constituée de 14 directeurs commerciaux travaillant dans de grandes entreprises appartenant à des secteurs d'activité variés (environnement, produits d'hygiène, pharmacie, informatique, bureautique, transports, spiritueux, etc.) et où il existe une procédure budgétaire clairement établie. Les cibles généralement visées par les chercheurs du courant RAPM sont les responsables de centres de coûts (les directeurs de production, le plus souvent) ou de centres de profit (Strategic Business Units). Nous avons choisi des responsables de la fonction commerciale pour les raisons suivantes :

- Premièrement, nous souhaitions avoir un échantillon relativement homogène de managers ayant des responsabilités comparables et gérant les mêmes types de budgets. La fonction de «responsable de centre de profit» peut recouvrir parfois des réalités disparates (Ardoin, 1995). Toutes les personnes rencontrées travaillent dans la fonction commerciale et y occupent une responsabilité élevée.

- Deuxièmement, les directeurs commerciaux ont des objectifs de nature claire. A l'opposé des centres de coûts dits discrétionnaires pour lesquels la fixation des objectifs et l'évaluation des performances est souvent problématique (Malleret, 1993), il est généralement admis que les directeurs commerciaux sont responsables d'un budget de chiffre d'affaires et d'un budget de dépenses commerciales. Ils ont donc des objectifs de chiffre d'affaires sur lesquels ils s'engagent et sont tenus de respecter une enveloppe de frais.

- Troisièmement, la fonction de directeur commercial se trouve généralement placée à un niveau intermédiaire de management, entre une hiérarchie (par exemple, la direction générale) et des collaborateurs (par exemple, des directeurs régionaux). Nous avons choisi de nous focaliser sur cette fonction afin de pouvoir analyser les phénomènes de pression budgétaire dans leur " épaisseur organisationnelle », c'est-à-dire en prenant en compte les inter relations entre les différents niveaux de management, le long de la chaîne hiérarchique.

- Quatrièmement, l'établissement du budget des ventes (objectifs de chiffre d'affaires) est généralement le point de départ de l'articulation budgétaire (Gervais, 2000 ; Giraud et al., 2002). De la pertinence du budget des ventes dépend souvent celle des autres budgets de l'entreprise (budgets de production, budgets des achats, etc.). C'est pourquoi, la fixation et l'atteinte des objectifs de chiffre d'affaires constituent un enjeu majeur dans les entreprises. Par conséquent, il nous semble pertinent d'observer les phénomènes de pression budgétaire au niveau d'une fonction - les directions commerciales - où la probabilité qu'ils se manifestent est la plus forte.

Enfin, un dernier critère a orienté la sélection de nos interlocuteurs : la qualité pressentie des échanges. Les sujets abordés, par définition délicats (pression budgétaire, stratégies de protection développées, etc.), exigeaient de pouvoir s'entretenir de manière ouverte et sincère avec nos interlocuteurs. C'est pourquoi, chacun des 14 directeurs commerciaux que nous avons rencontrés connaissait personnellement l'un des trois chercheurs de l'équipe (ou une personne de son entourage). Ce processus de sélection, susceptible d'induire certains biais méthodologiques, a néanmoins été privilégié afin, en un unique d'entretien, de disposer de la 
confiance des interlocuteurs. Il convient également de noter que nous nous sommes engagés à une confidentialité totale qu'il s'agisse de nom, de fonction ou d'entreprise.

\subsection{Le recueil des données}

Celui-ci s'est effectué au travers d'entretiens semi-directifs d'une durée moyenne d'une heure et demie. Nous ne souhaitions pas avoir recours à des questionnaires fermés comme ceux utilisés par les chercheurs du courant RAPM, mal adaptés à une compréhension fine des mécanismes et des « variables » mobilisées dans ces recherches. A l'autre extrême, l'étude de cas unique ne nous paraissait pas non plus adaptée. D'une part, elle exige une immersion dans l'entreprise qui attend, en retour, un feed-back. Compte tenu des objectifs de notre recherche, il était important que nos interlocuteurs se sentent libres de s'exprimer, en sachant que leurs propos ne seraient en aucune manière rapportés en interne. D'autre part, le recueil des données dans plusieurs entreprises autorise des observations à partir d'une base empirique plus large que les études de cas approfondies. Ainsi, le dispositif de recueil d'informations choisi permet d'appréhender des phénomènes complexes, portant sur le «comment» et le « pourquoi », dans un domaine clairement délimité (Eisenhart, 1989; Ahrens et Dent, 1998), et d'expliquer des résultats contradictoires ou ambigus produits par les précédentes recherches dans le domaine (Lillis et Mundy, 2003).

Notre objectif était moins de recueillir des données factuelles sur les procédures budgétaires que de comprendre les perceptions qu'en ont les managers. A cet effet, nous avons associé au guide d'entretien l'utilisation de visuels. Le recours à ces derniers s'inscrit dans les techniques dites projectives. Issues du champ de la psychologie, les techniques projectives ont été adaptées aux recherches marketing à partir de 1950 (Haire, 1950). Au fil des années, elles sont devenues un complément indispensable aux études de motivation menées auprès de consommateurs (Anzieu et Chabert, 1983 ; Guelfand, 1998).

Ces techniques n'ont pas, à notre connaissance, été mobilisées dans les recherches en contrôle de gestion. L'entretien, réalisé à partir d'un guide d'entretien semi-directif, abordait successivement les thèmes suivants : l'environnement organisationnel et externe, l'élaboration et la négociation des objectifs budgétaires, le suivi des résultats en cours d'année, l'évaluation finale et les liens avec les systèmes de récompense. Nous avons fait réaliser par un professionnel quatre jeux de visuels illustrant différentes attitudes possibles au fil de ces étapes. L'annexe 1 indique la finalité de chaque série de visuels et l'annexe 2 présente, à titre d'illustration, les visuels constitutifs des séries B et D. Chaque manager interviewé avait été averti par le document présentant l'étude qu'il pourrait avoir à "réagir à des visuels ». Ceci était de nouveau rappelé lors de l'introduction de l'entretien. L'entretien faisait d'abord l'objet d'un déroulement classique : le manager présentait son organisation, l'environnement dans lequel il évoluait et ses missions principales. Puis, sur les aspects budgétaires, la plupart des étapes-clés étaient introduites par une série de visuels qui l'obligeaient à réagir de façon spontanée à un stimulus extérieur à son organisation professionnelle. Il faisait ensuite le lien avec sa perception des processus budgétaires auxquels il était confronté.

En réalité, les visuels exigeaient de la part du manager un effort d'interprétation. Certains de nos interlocuteurs s'y retrouvaient plus facilement que d'autres. Dans tous les cas, cependant, les étapes au cours desquelles le manager a réagi face aux visuels ont constitué des moments forts de l'entretien en permettant au manager de libérer sa parole et de nous donner un 
éclairage sur son vécu du processus budgétaire. Ceci aurait été plus difficile à obtenir dans un temps limité sans technique projective.

Chaque entretien a été enregistré et a fait l'objet d'une retranscription exhaustive (18 à 25 pages A4 interligne simple par entretien). Leur anonymat et celui de l'entreprise ayant été garantis, les managers se sont exprimés très librement.

Afin d'organiser les données obtenues, nous nous sommes notamment appuyés sur les variables mises en avant par les travaux de recherche antérieurs.

\subsection{L’analyse des données}

Le contenu d'entretiens semi-directifs, tels que ceux que nous avons menés, permet deux types d'analyse. L'une, dénommée « analyse verticale » s'intéresse à la logique personnelle de l'individu; l'autre, "l'analyse horizontale », étudie la façon dont un thème donné est traité par les différentes personnes interviewées. Nous nous sommes essentiellement appuyés sur l'analyse horizontale pour ce travail. Dans la partie 4, nous présentons les résultats concernant les thèmes suivants : la pression mise sur le respect du budget (budget emphasis ou RAPM), le degré de participation au processus budgétaire et les effets sur les comportements.

La grille ci-dessous met en relation chaque étape de l'entretien avec les thèmes explorés :

Tableau $\mathbf{n}^{\circ} 1$

Thèmes explorés pour chaque série de visuels

\begin{tabular}{|l|c|c|c|}
\hline & $\begin{array}{c}\text { Pression mise sur le } \\
\text { respect du budget }\end{array}$ & $\begin{array}{c}\text { Participation au } \\
\text { processus budgétaire }\end{array}$ & $\begin{array}{c}\text { Effets sur les } \\
\text { comportements }\end{array}$ \\
\hline Série A & $\mathrm{X}$ & & \\
\hline Série B & & $\mathrm{X}$ & \\
\hline Série C & $\mathrm{X}$ & $\mathrm{X}$ & $\mathrm{X}$ \\
\hline Série D & $\mathrm{X}$ & & $\mathrm{X}$ \\
\hline
\end{tabular}

Pour chacun des thèmes examinés, nous nous sommes attachés à faire ressortir les positions et opinions exprimées par le plus grand nombre de managers. Nous indiquerons les pourcentages approximatifs de managers ayant exprimé tel ou tel point de vue, en insistant cependant sur le fait qu'ils ne constituent que des ordres de grandeur n'ayant aucune validité statistique.

\section{Les résultats}

L'analyse des entretiens effectués nous a permis de mieux comprendre les formes que prend la pression budgétaire ainsi que ses liens avec le degré de participation budgétaire. Enfin, nous examinons les effets sur les comportements, tels qu'ils apparaissent dans les entretiens. 


\subsection{La pression budgétaire}

Dans la littérature RAPM, une situation de forte pression budgétaire se caractérise par une combinaison des trois éléments suivants :

a) Le manager est personnellement et individuellement tenu de respecter des objectifs budgétaires.

b) Ces objectifs sont exprimés en termes exclusivement financiers (chiffre d'affaires, coûts, profits).

c) En fin d'année, le seul critère pris en compte pour évaluer les performances du manager est le respect de ses objectifs budgétaires. Des facteurs plus qualitatifs ou subjectifs (niveau d'objectifs difficilement atteignable, importance de l'effort fourni par le manager, performance du manager sur d'autres dimensions) sont ignorés.

La situation de pression budgétaire telle qu'elle est envisagée traditionnellement par la littérature se manifeste donc essentiellement en fin de période, lors de l'évaluation des performances. De plus, elle met en scène un individu isolé, le manager, confronté à un supérieur hiérarchique qui joue le rôle de l'évaluateur.

Nos entretiens nous ont permis de mettre à jour une réalité plus complexe. D'une part, la pression budgétaire constitue un processus s'exerçant de manière continue et susceptible de prendre diverses formes à différents moments de l'année. D'autre part, dans certaines entreprises, la logique individuelle qui caractérise les situations de pression budgétaire cède la place à une gestion plus collective de cette dernière.

\subsubsection{La pression budgétaire : un processus continu}

Pour l'ensemble des managers rencontrés, la pression budgétaire s'exerce tout au long du processus allant de la négociation des objectifs à l'évaluation des performances.

La perception qu'un manager aura de la pression budgétaire commence à se constituer dès la phase même d'établissement de ses objectifs budgétaires. A cette étape, deux facteurs jouent un rôle décisif : son degré de participation aux discussions et la difficulté des objectifs fixés.

"Oui, je perçois une forte pression: les objectifs sont très ambitieux. Cette année par exemple, j'ai présenté un plan et on m'a dit : c'est très bien mais il faudrait tant de plus. J'ai dit: OK on affiche tant de plus mais pour l'instant je ne sais pas comment je vais le faire» (Directeur commercial - Secteur de l'agro-alimentaire).

Un responsable percevra une pression budgétaire moindre s'il a le sentiment de pouvoir exercer une influence sur le niveau des objectifs fixés pour son entité. Ainsi que nous le détaillerons par la suite, la pression budgétaire et la participation budgétaire sont reliées.

De plus, lorsque le manager a le sentiment que les objectifs à atteindre sont excessivement difficiles, il percevra une plus forte pression budgétaire. Certains chercheurs ont exploré l'effet de la difficulté perçue des objectifs sur la motivation (Kenis, 1979) ou sur la performance (Stedry, 1960 ; Hofstede, 1967) mais les liens entre difficulté des objectifs et pression budgétaire ont été peu analysés.

Ensuite, la pression budgétaire peut être renforcée ou atténuée lors des reprévisions en cours d'année : est-il possible de revenir sur les objectifs fixés en début d'exercice ? 
"Si les remarques de mes collaborateurs s'avèrent fondées (cas de collaborateurs considérant leurs objectifs de chiffre d'affaires comme trop élevés), j'essaye de négocier un éventuel réajustement avec la direction générale en cours d'année, lors des revues de compte »(Directeur commercial - Secteur des Télécommunications).

Enfin, et nos résultats confirment en ce point la littérature RAPM, la pression budgétaire perçue par les managers dépend du couplage plus ou moins strict entre le système de récompenses et le degré d'atteinte des objectifs budgétaires : le non respect des engagements budgétaires se traduit-il par une sanction forte ou, au contraire, la hiérarchie prend-elle en compte des éléments plus qualitatifs lors de l'évaluation des performances (événements imprévus non maîtrisables, difficulté élevée des objectifs, etc.)?

"Si quelqu'un (un collaborateur) n'a pas atteint ses objectifs mais que je sens qu'il a fait quand même le maximum, j'ai une marge de manœuvre en termes de rémunération: $30 \%$ de la prime est liée à l'atteinte d'objectifs qualitatifs. C'est ce que j'ai en dernier ressort» (Directeur commercial-Secteur des Télécommunications).

Pour comprendre les phénomènes de pression budgétaire, il est nécessaire d'examiner comment celle-ci s'exerce tout au long de l'année, alors que les chercheurs du courant RAPM ont focalisé leur attention sur la phase « évaluation des performances en fin d'année ».

Par ailleurs, ce processus se reproduit chaque année. Généralement, les mêmes acteurs sont impliqués durant plusieurs années consécutives. Ce qui s'est passé lors des exercices antérieurs influence aussi alors le degré de pression budgétaire. Ainsi, des relations de confiance se développent dans certains cas :

"Sur les trois dernières années, on a fait nos objectifs. Comme on ne se plante pas dans les bottom-up, l'organisation nous fait confiance et la direction n'en rajoute pas trop » (Directeur Commercial - Secteur Pharmacie).

\subsubsection{Une pression budgétaire parfois plus collective qu'individuelle}

La littérature RAPM postule des responsabilités strictement délimitées et une évaluation des performances uniquement individuelle. Près de $70 \%$ des managers décrivent une gestion plus collective de l'atteinte des objectifs dans le contexte commercial. Dans certains cas, les objectifs collectifs sont plus importants que les objectifs individuels :

" Auparavant, chaque personne avait un objectif de prise de commande individuel. On s'est rendu compte que les objectifs étaient trop individualisés et ne privilégiaient pas assez l'équipe .... Dans notre secteur, les processus de négociation sont relativement longs ; les cycles de vente dépassent l'année; les objectifs, eux, sont annuels. On joue sur la masse, souvent certaines affaires se décalent; il y en a d'autres qui vont avoir lieu pendant l'année, d'où, sur la population commerciale et marketing, un objectif de prise de commande collectif » (Directeur Commercial - Secteur des Biens d'équipement).

Par ailleurs, les objectifs peuvent être revus à la hausse en cours d'année pour certaines entités, s'il s'avère que d'autres entités connaissent des difficultés et n'atteindront pas les 
objectifs qui leur avaient été initialement fixés. S'instaurent alors des mécanismes proches de la mutualisation que nous avons rencontrés dans la plupart des entreprises interrogées.

"L'important c'est que le groupe respecte ses objectifs globaux, il y a des réajustements. Si une entité a eu des difficultés commerciales, de gestion opérationnelle ou peu importe, on peut être amené à demander aux autres de faire un effort pour compenser .... Cela s'est déjà produit » (Directeur Commercial - Secteur de l'Environnement)

«Au moment où on finalise le plan, en septembre, si on s'aperçoit que pour telle ou telle raison un autre marché ne fonctionne pas aussi bien que prévu, on peut me demander d'en faire plus .....

Ensuite, en cours d'année, au moment du R2 (reprévision en milieu d'année), on (sa hiérarchie) peut me dire: votre voisin (responsable d'une autre zone géographique), il a tellement bien réussi que vous avez moins de pression - ou par contre: la pression est confirmée ou même doublée parce que le voisin ne va pas faire ses objectifs " (Directeur Commercial - Secteur agro-alimentaire).

Cette dimension collective se manifeste également lors de l'évaluation des performances :

"Chez nous, les possibilités d'évolution sont limitées. Les gens ne se battent pas pour émerger par rapport aux autres. Je trouve que cela crée une espèce de solidarité, d'esprit d'équipe. Ici, la reconnaissance, elle est plus globale; c'est pas l'individu, elle est collective » (Directrice Commerciale - Secteur de l'Hygiène).

"Si l'objectif de la société est atteint, tout le monde aura accès à une prime personnelle. Si l'objectif n'est pas là, c'est zéro pointé pour tout le monde " (Directeur commercial-Secteur de l'emballage).

Lorsque la pression est davantage placée sur l'atteinte d'objectifs collectifs, les managers perçoivent une moindre pression au niveau individuel. Certes, il peut leur être "demandé plus » pour compenser les performances médiocres d'une autre entité mais, en contrepartie, cette gestion collective de la pression développe une plus grande solidarité : c'est l'atteinte des objectifs globaux de rentabilité pour les actionnaires qui importe en dernière instance.

\subsection{Pression budgétaire et participation budgétaire}

La participation budgétaire traduit la possibilité pour les managers d'intervenir dans la fixation de leurs objectifs budgétaires. La littérature RAPM s'est focalisée sur la relation exclusive entre le manager et son supérieur hiérarchique direct sans s'intéresser aux autres niveaux hiérarchiques avec lesquels le manager est en interaction.

Nos données montrent que le degré de participation budgétaire doit être étudié en examinant les relations le long de la ligne hiérarchique. Les directeurs commerciaux interrogés se situent, dans leur majorité, au point de rencontre de deux contraintes : satisfaire les objectifs globaux de chiffre d'affaires imposés par leur hiérarchie (dans leur cas, la direction générale) et prendre en compte les informations et problèmes remontés du terrain par leurs collaborateurs. 
"La pression budgétaire top-down, elle est au-dessus de moi. Il y a ma hiérarchie à qui on dit: La France, l'année prochaine, il faudra qu'elle sorte un chiffre comme ça. Lui (son supérieur, le directeur général), il voit ce que je peux décemment faire et il voit ce que tous les autres peuvent décemment faire. Et moi, je sais ce que mes collaborateurs peuvent faire. Si je leur mets des objectifs inatteignables, c'est pas très motivant. C'est là où la rencontre est parfois difficile » (Directeur Commercial - Secteur de la pharmacie)

\subsubsection{Des objectifs globaux imposés}

Près de trois-quarts des directeurs commerciaux décrivent des situations dans lesquelles les objectifs globaux (notamment chiffre d'affaires global à réaliser) leur sont largement imposés. Ces objectifs résultent des engagements pris par leur hiérarchie vis-à-vis des actionnaires.

"Il est perdu d'avance de tenter de contester les objectifs de là-haut. Cela ne changera rien, cela ne changera rien .... Je répartis les objectifs commerciaux entre mes différentes zones géographiques après discussion avec mes équipes et puis je présente cela à mon patron. Lui, cela lui est égal. Je dois me débrouiller comme je peux et, ce qu'il veut, c'est qu'à la fin de l'année, les résultats soient là ! (Directeur Commercial - Secteur des Télécommunications).

"Très vite, on a un point de départ assez clair en début d'année ... les objectifs financiers sont annoncés au marché .... donc ces objectifs après, il faut bien les décliner, il faut bien arriver à les respecter ... bien sûr les entités opérationnelles ont leur mot à dire mais au global en consolidé, si la somme des objectifs des divisions n'est pas conforme à ce qu'on avait annoncé, ça redescend ... » (Directeur Commercial-Secteur de l'Environnement).

Les directeurs commerciaux doivent ensuite répartir cet objectif global entre leurs différents responsables produits ou marchés. La démarche est alors généralement plus participative.

\subsubsection{Un management de proximité}

Lorsque le directeur commercial va à son retour décliner son objectif global de chiffre d'affaires en objectifs locaux par zone géographique, par ligne de produit, etc., il adopte une démarche plus participative. Il lui faut écouter ses collaborateurs afin de prendre en compte les réalités du terrain. Une très forte majorité de managers ont ainsi indiqué qu'ils privilégiaient une approche bottom-up pour déterminer les objectifs de leurs collaborateurs. Au cours de celle-ci, la discussion ne porte pas uniquement sur les objectifs mais aussi sur les plans d'action envisagés. Les directeurs commerciaux développent un management de proximité. L'établissement des budgets résulte d'un processus fortement itératif où les discussions portent sur des points précis et opérationnels : quels sont les différents scénarios envisageables? Quelles retombées est-on en droit d'attendre de chacune des actions envisagées?

"Plus on se rapproche d'un marché, plus c'est itératif, on descend beaucoup plus au fond des choses pour dire très précisément et très concrètement comment on va faire, à quoi correspondent les objectifs» (Directeur Commercial - Secteur de l'Environnement).

"Quand on travaille sur les budgets avec mes chefs de zone, il y a une vraie discussion. Je leur demande de m'expliquer pourquoi ils estiment que telle orientation est meilleure que 
telle autre action ... à la fin, on arrive toujours à se mettre d'accord » (Directeur Commercial - Secteur des spiritueux).

En réalité, les discussions n'interviennent pas uniquement au moment de l'établissement des budgets. Les échanges se déroulent de manière continue tout au long de l'année.

«Les discussions n'interviennent pas qu'au moment de l'établissement des objectifs, elles interviennent aussi avant, très clairement. C'est un dialogue un peu continuel. On n'attend pas la formalisation du plan et du budget pour discuter des choses » (Directeur Commercial - Secteur agro-alimentaire).

C'est dans le contexte de cette double dynamique top-down et bottom-up, que se situe la pression budgétaire.

\subsubsection{Les influences réciproques entre participation budgétaire et pression budgétaire}

Les liens entre participation budgétaire et pression budgétaire sont complexes. Lorsque les directeurs commerciaux sont confrontés à une forte pression budgétaire émanant de leur hiérarchie, ils tendent à multiplier les échanges avec leurs équipes (participation budgétaire) de manière à disposer d'un maximum d'informations à la fois au moment où ils négocient les objectifs mais également dans le suivi en cours d'année des réalisations. Par ailleurs, quand ils ont le sentiment de pouvoir dialoguer (discussions, consultations) facilement avec leur propre hiérarchie, c'est-à-dire généralement la direction générale, ils perçoivent une moindre pression budgétaire.

"Quelque soit l'autonomie que je peux avoir, je donne un degré de visibilité à ma hiérarchie ce qui fait qu'elle devient solidaire de ce que je fais ... Tout au long de l'année, je prends mes décisions, j'en parle à ma hiérarchie, et il y a une discussion qui peut s'instaurer ... On est solidaire dans le fait d'atteindre les objectifs et on est solidaire dans le fait de ne pas les atteindre» (Directeur Commercial - Secteur des services informatiques).

Les situations décrites ci-dessus mettent en scène des directeurs commerciaux aux prises avec des obligations en partie contradictoires : accepter les objectifs fixés par la direction générale et prendre en compte les contraintes mises en avant par leurs collaborateurs au moment où ils établissent les objectifs de ces derniers. De plus, pour une majorité d'entre eux, une partie souvent conséquente de leur rémunération dépend ensuite de l'atteinte des objectifs définis lors du processus budgétaire. Parmi les managers rencontrés dans le cadre de cette recherche, plusieurs ont reconnu développer des stratégies pour éviter une trop forte pression budgétaire ou y faire face, le cas échéant.

\subsection{Les effets de la pression budgétaire : création de slack et orientation sur le court terme.}

Selon les travaux du courant RAPM, les managers tendraient à développer des comportements négatifs lorsque l'évaluation de leur performance dépend strictement du respect de leur budget. Nos résultats montrent que la pression budgétaire perçue par les managers ne résulte pas uniquement du couplage plus ou moins strict entre le respect du budget et l'attribution de récompenses (ou de sanctions) mais dépend aussi des phases amont 
au cours desquelles les objectifs sont négociés et les résultats suivis durant l'année. En réalité, dans leur majorité, les directeurs commerciaux acceptent et comprennent que le respect des objectifs budgétaires constitue un élément central de l'évaluation de leurs performances. Cette logique contractuelle (Ouchi, 1980) est généralement bien admise. Ce que critiquent parfois certains de nos interlocuteurs, ce sont les modalités suivant lesquelles le contrat est établi. Lorsque les objectifs sont considérés comme difficiles à atteindre et comme relativement imposés par la hiérarchie, la pression budgétaire est alors perçue comme forte et ceci favorise le développement de deux types de comportements. D'une part, les managers tendent à développer du slack pour se prémunir contre le risque de ne pas atteindre les objectifs. D'autre part, ils sont conduits à prendre des décisions qui privilégient les résultats à court terme tout en étant conscients que, dans certains cas, ces décisions sont contraires à la compétitivité à moyen et long terme de leur entreprise.

\subsubsection{La création de slack}

Le slack se constitue au moment de la détermination des objectifs. Les travaux du courant RAPM ont examiné une forme donnée de slack: celui créé par un manager quand il négocie avec sa hiérarchie. Près de la moitié de nos interlocuteurs ont ainsi reconnu développer des manœuvres destinées à se constituer des réserves. En particulier, lorsque des clients ou des marchés présentent des risques importants, les responsables commerciaux s'efforcent de sousévaluer le chiffre d'affaires qu'ils estiment pouvoir faire avec des clients ou des marchés plus sûrs de manière à "se couvrir en cas de problème ". Le principal motif avancé par nos interlocuteurs pour justifier ces pratiques est la pression budgétaire à laquelle ils sont soumis.

"Avec cette très forte pression, la tendance est de dire : il faut se réserver quelque chose, ne pas engager tout ... Si je pense faire 100, je vais présenter 95 ... quand on élabore les plans, on a toujours tendance à être un peu en dessous " (Directeur Commercial - Secteur agroalimentaire).

"J'étais certaine qu'avec le client $X$, cela se passerait bien. J'ai dit: je vais progresser de +10 sachant que j'avais un potentiel de progresser de +20 . Mais j'ai dit +10 parce que je sais qu'il y a toujours un autre client avec lequel on n'atteindra pas nos objectifs » (Directrice commerciale - Produits d'Hygiène)

Par ailleurs, un deuxième type de slack a été détecté dans notre étude : celui développé par la ligne hiérarchique lors du déploiement des objectifs. De manière spontanée, près d'un tiers des responsables commerciaux ont dénoncé un système de prise de marges de manœuvre descendantes par leurs supérieurs hiérarchiques. Du fait de cette pratique, les directeurs commerciaux se trouveraient confrontés à des objectifs excessivement difficiles à réaliser.

"Au départ, c'est le comité exécutif qui va définir ce que devront être les objectifs commerciaux globaux au niveau international et ensuite on a une redistribution qui se fait au niveau des différentes entités. Il y a toujours un monsieur Plus qui en rajoute un peu. Et en fait, quand cela arrive au niveau des commerciaux, c'est toujours la même chose: ils se plaignent que c'est inatteignable» (Directeur Commercial - Entreprise de Télécommunications). 
"Les objectifs globaux sont fixés au départ avec un certain niveau et ensuite, tous les intermédiaires en management prennent la sécurisation. Il faut le savoir ! Et le commercial in fine, il prend tout en plus sur les épaules et le plus, il est énorme » (Directeur CommercialSociété de services informatiques).

Si les phénomènes de constitution de slack prennent place au moment de l'établissement des objectifs, un autre effet de la pression budgétaire intervient, quant à lui, en cours d'année : il s'agit de l'orientation des décisions.

\subsubsection{La tentation du court terme}

Un peu moins de la moitié de nos interlocuteurs ont reconnu que, face à une forte pression budgétaire, ils sont parfois conduits à prendre des décisions permettant de « tenir » le budget tout en reconnaissant qu'elles peuvent aller à l'encontre de l'intérêt à plus long terme de l'entreprise.

"Très clairement, le risque avec cette volonté d'afficher de bons résultats financiers, avec cette pression, c'est d'aller à l'encontre de la politique à long terme de l'entreprise .... Pour pouvoir atteindre mon objectif de contribution, les solutions c'est de ne pas investir dans tel pays, de supprimer telle campagne de promotion, de reporter des projets. Cela ne changera rien sur mes ventes de l'année, ça c'est clair. Mais dans trois, quatre ans? " (Directeur Commercial - Secteur agro-alimentaire).

"Si les ventes ne sont pas au rendez-vous, je ne vais pas dépenser tout le budget. Ceci dit, c'est un peu malthusien, car .... C'est un moyen au minimum d'atteindre le résultat opérationnel, même si le chiffre d'affaires n'est pas au rendez-vous » (Directeur Commercial - Secteur de l'emballage).

Ainsi, les résultats de notre recherche montrent que les managers sont parfois contraints de développer des manœuvres afin de faire face à la pression budgétaire. Ces manœuvres prennent place au moment de l'élaboration des objectifs mais également en cours d'année.

\subsection{Synthèse des résultats}

Notre étude a pris comme point de départ les recherches du courant RAPM et a tenté de mieux comprendre des variables importantes mobilisées par ces travaux - pression budgétaire, participation budgétaire - ainsi que leurs articulations et leurs effets.

Le tableau ci-dessous présente une synthèse de nos résultats et les met en perspective par rapport à ceux issus du courant RAPM. 
Tableau $n^{\circ} 2$

Synthèse et mise en perspective des résultats de notre étude par rapport à ceux du courant RAPM

\begin{tabular}{|c|c|c|}
\hline $\begin{array}{l}\text { Les concepts et leur } \\
\text { articulation }\end{array}$ & Les travaux sur la RAPM & $\begin{array}{l}\text { Les apports de notre étude } \\
\text { exploratoire }\end{array}$ \\
\hline $\begin{array}{l}\text { La pression budgétaire: } \\
\text { à quel moment } \\
\text { l'observe-t-on? }\end{array}$ & $\begin{array}{l}\text { Evaluation des performances } \\
\text { en fin d'année }\end{array}$ & Un processus continu \\
\hline $\begin{array}{l}\text { La pression budgétaire : } \\
\text { comment s'exerce-t- } \\
\text { elle? }\end{array}$ & Pression individuelle & Pression individuelle et collective \\
\hline $\begin{array}{l}\text { La participation } \\
\text { budgétaire }\end{array}$ & $\begin{array}{l}\text { Le manager a la possibilité de } \\
\text { participer à la fixation de ses } \\
\text { objectifs dans le cadre d'un } \\
\text { dialogue avec son supérieur } \\
\text { hiérarchique }\end{array}$ & $\begin{array}{l}\text { Elle doit être examinée en } \\
\text { considérant les inter-relations } \\
\text { entre les différents niveaux } \\
\text { hiérarchiques. }\end{array}$ \\
\hline $\begin{array}{l}\text { Les relations entre } \\
\text { participation budgétaire } \\
\text { et pression budgétaire }\end{array}$ & $\begin{array}{l}\text { La participation budgétaire } \\
\text { atténuerait les effets négatifs } \\
\text { sur les comportements induits } \\
\text { par la pression budgétaire } \\
\text { (résultats non totalement } \\
\text { convergents, cf. partie 1) }\end{array}$ & $\begin{array}{l}\text { La pression budgétaire conduit } \\
\text { les directeurs commerciaux à } \\
\text { accroître la participation } \\
\text { budgétaire } \\
\text { collaborateurs. } \\
\text { Lorsqu'ils ont eux-mêmes la } \\
\text { possibilité de discuter de leurs } \\
\text { objectifs avec leur hiérarchie, ils } \\
\text { perçoivent une moindre pression } \\
\text { budgétaire. }\end{array}$ \\
\hline $\begin{array}{l}\text { Les effets de la pression } \\
\text { budgétaire }\end{array}$ & $\begin{array}{l}\text { effets négatifs sur les } \\
\text { comportements : création de } \\
\text { slack, orientation court terme } \\
\text { et manipulation des données } \\
\text { (résultats non totalement } \\
\text { convergents) }\end{array}$ & $\begin{array}{l}\text { Notre étude confirme ces } \\
\text { résultats, en mettant en évidence } \\
\text { deux conséquences possibles de } \\
\text { la pression budgétaire : la } \\
\text { création de slack et l'orientation } \\
\text { court terme. } \\
\text { Mise en évidence des } \\
\text { phénomènes de slack descendant. }\end{array}$ \\
\hline
\end{tabular}

Les travaux du courant RAPM se sont généralement focalisés sur une étape donnée du processus de contrôle et plus particulièrement sur l'évaluation des performances en fin d'année. Or, le processus de contrôle, dans lequel s'insère le budget, est continu. La phase d'identification des objectifs et d'allocation des ressources est suivie d'une phase d'observation des actions en cours et enfin d'une phase de comparaison des résultats avec les prévisions (Bouquin, 1999). Notre étude indique que le degré de pression budgétaire perçu par les managers ne dépend pas uniquement de la manière dont sont évaluées leurs performances en fin d'année mais relève d'un processus dynamique et continu au fil des différentes phases. 
Par ailleurs, si la pression budgétaire est parfois ressentie au niveau individuel, nous avons pu observer certains phénomènes de solidarité. Dans ces entreprises, les managers ne se perçoivent pas comme des individus isolés face à leurs objectifs ; ils savent qu'ils participent à l'atteinte d'objectifs plus globaux; la pression budgétaire est en quelque sorte partagée. Ces résultats confirment les analyses de chercheurs français: la mise en place d'une gestion budgétaire couplée à des rétributions pécuniaires individuelles et à une relation supérieur/subordonné fondée sur un contrat s'inscrit dans le modèle culturel américain (Gervais, 2000, p. 546). Ce modèle d'animation n'est pas universel (Segal, 1987 ; d'Iribarne, 1989 ) et nos résultats montrent qu'il peut subir des amendements. Il n'existe pas de système de contrôle budgétaire unique et valable pour toutes les entreprises (Berland, 2002). En l'occurrence, la plupart des managers rencontrés dans le cadre de cette étude perçoivent une pression budgétaire forte et qui parfois s'intensifie. Cependant, cette pression n'est pas uniquement gérée dans le cadre d'une relation contractuelle entre un supérieur et un subordonné.

Par ailleurs, l'examen de la pression budgétaire est indissociable de celui des processus de participation budgétaire. En cela, nos travaux confirment certains résultats obtenus par des chercheurs du courant RAPM. Les liens entre pression budgétaire et participation budgétaire sont complexes. En effet, lorsque le manager est confronté à une forte pression budgétaire, il tend à accroître le degré de participation budgétaire, de manière notamment à travailler plus étroitement avec ses équipes. Par ailleurs, lorsque le manager a l'opportunité de faire entendre son point de vue lors de la négociation de ses objectifs budgétaires avec sa hiérarchie, il perçoit une moindre pression budgétaire. Ces résultats mettent en lumière les modalités suivant lesquelles les dispositifs du contrôle de gestion contribuent à développer un processus de dialogue le long de la ligne hiérarchique (Fiol et Lebas, 1999).

Enfin, notre étude confirme certains effets négatifs de la pression budgétaire : le développement de slack et la tendance à prendre des décisions à court terme. Une observation intéressante est que le slack se constitue de manière ascendante mais procède aussi parfois d'une logique descendante (prise de marges de sécurité par la hiérarchie).

Le schéma ci-dessous tente de rendre compte des principales inter relations que notre recherche a contribué à mettre en lumière :

Figure 2

Facteurs à l'origine de la pression bugétaire et comportements induits

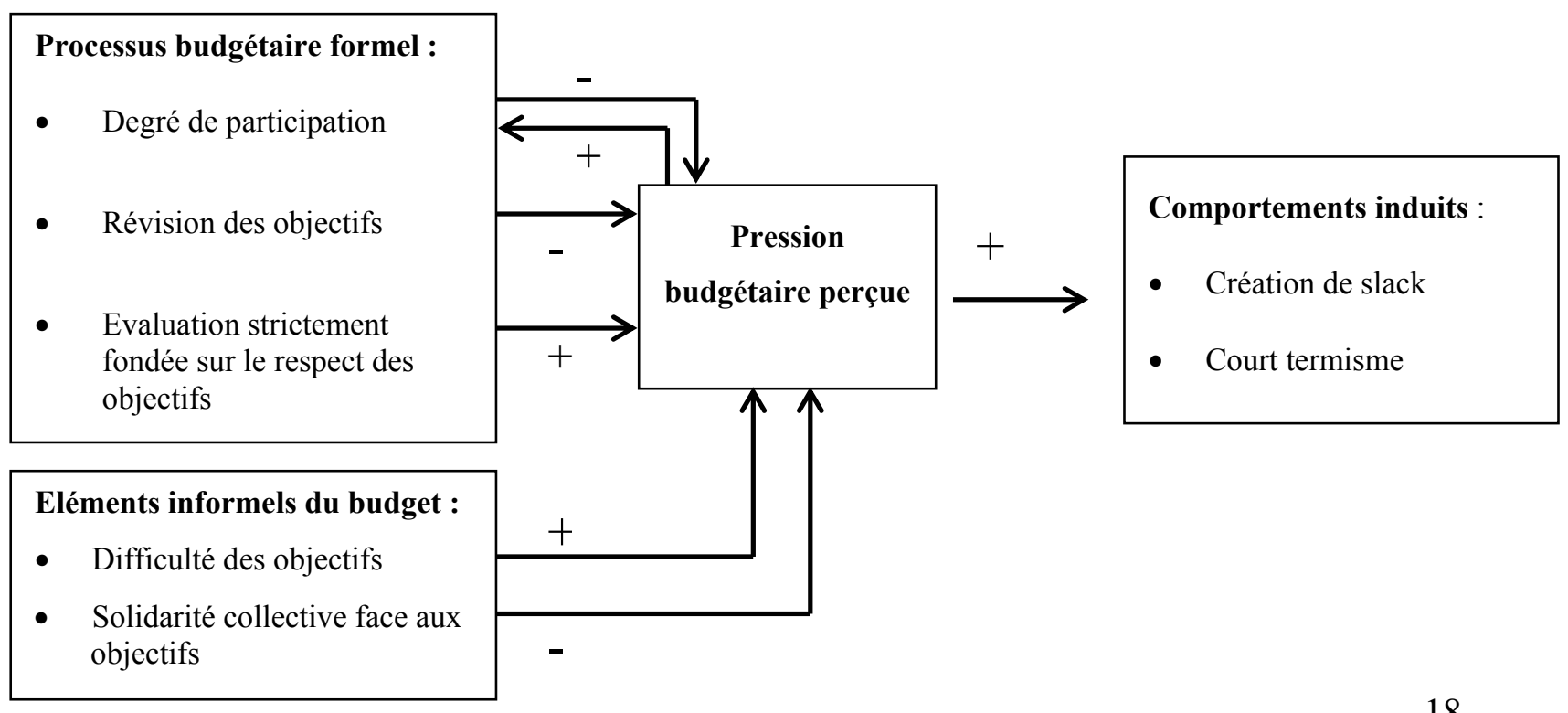


En définitive, la pression budgétaire perçue par le manager résulte du poids relatif de différents facteurs qui exercent des influences contradictoires. Certains facteurs sont inscrits dans la procédure budgétaire : la possibilité ou non pour les managers de participer à la fixation des objectifs, la révision des objectifs en cours d'exercice, l'existence de règles couplant strictement le respect du budget et l'évaluation des performances. D'autres facteurs sont de nature plus informelle: la difficulté perçue des objectifs et les phénomènes de solidarité.

\section{Conclusion}

La gestion budgétaire a fait l'objet de nombreuses critiques au cours de ces dernières années (Hope et Fraser, 2000 ; Berland, 2001 ; Berland et Pinçon, 2002). On a notamment reproché au budget son incapacité à jouer tous les rôles qu'on lui attribue. Cependant, des enquêtes récentes ont également indiqué que la préparation et le suivi du budget constituent toujours un dispositif central dans lequel les responsables opérationnels sont même de plus en plus impliqués (Jordan, 1998 ; Guizani et Brunhes-Faure, 2002). Une étude sur les rôles actuels de la gestion budgétaire en France conclut que le maintien de la gestion budgétaire tient plus à ses rôles humains, comme par exemple l'orientation des comportements, qu'économiques (Gignon-Marconnet, 2003). Dès lors, les mécanismes suivant lesquels les objectifs budgétaires sont établis, suivis et finalement utilisés pour l'évaluation des performances nous semblent mériter un examen plus approfondi.

Les travaux menés par les chercheurs du courant RAPM ont servi de point de départ à notre recherche. Bien que nombreux et inscrits dans un courant de recherche clairement identifié, ces travaux ne sont pas parvenus à dégager des résultats concluants. Ce constat nous a conduit à opter pour une approche méthodologique différente s'appuyant sur des entretiens semidirectifs et sur le recours à des visuels (méthodes projectives). Nous avons ainsi pu mieux comprendre les modalités suivant lesquelles s'exercent la pression budgétaire et la participation budgétaire ainsi que leurs conséquences.

Il convient de souligner les limites de notre démarche de recherche. Les relations que nous avons identifiées devront être validées à partir d'observations sur un échantillon plus large. Par ailleurs, nous n'avons pas réalisé des études de cas approfondies mais nous avons simplement rencontré un seul interlocuteur, par entreprise. Nos résultats s'appuient donc sur l'analyse de perceptions uniquement individuelles.

Cependant, malgré ses limites, notre étude a permis de mieux appréhender les phénomènes organisationnels en jeu lors de la fixation des objectifs, de leur suivi et de l'évaluation des performances. Elle ouvre également des pistes pour analyser les liens parfois complexes qui lient la pression budgétaire, la participation budgétaire et leurs effets sur les comportements. En ce sens, nous espérons qu'elle contribuera à apporter un nouvel éclairage aux travaux du courant RAPM et qu'elle constituera un point de départ possible pour de nouvelles recherches. 


\section{Annexe 1 : Les séries de visuels}

Les deux premières séries de visuels (séries $\mathrm{A}$ et $\mathrm{B}$ ) illustraient des situations/attitudes dans la phase d'élaboration des budgets. La série $\mathrm{C}$ met en scène des situations en cours d'année et la série $\mathrm{D}$ porte sur l'évaluation en fin d'année.

La série $\boldsymbol{A}$ a été utilisée pour évoquer avec le manager ses sentiments quand il doit construire son budget (stress, ennui, sérénité, etc.).

La série B devait permettre d'explorer les relations entre le manager, sa hiérarchie et ses collaborateurs lors de l'établissement du budget. En particulier, nous avons cherché à cerner le degré de marge de manœuvre du manager dans ses négociations avec le niveau hiérarchique supérieur.

La série $\boldsymbol{C}$, permettait de faire réagir les managers sur les thèmes suivants : que se passe-t-il si un écart défavorable significatif apparaît en cours d'année? Se sent-il seul face au problème ou y a-t-il une prise en charge plus collective de celui-ci ? Dans quelle mesure est-il possible ou souhaitable qu'il en parle avec son supérieur hiérarchique? L'organisation autorise-t-elle une modification des objectifs en cours d'année?

La série $\boldsymbol{D}$ suggérait différents types de scénarios en cas de non atteinte des objectifs en fin de période : le non respect du budget conduit-il à des sanctions? D'autres éléments sont-ils pris en compte lors de l'évaluation du manager? Quelle est l'attitude de la hiérarchie ? L'échec est-il perçu comme collectif ou est-il attribué à un responsable? Quelles en sont les conséquences?

\section{Annexe 2 : Exemples de visuels}

\section{Série $B-n^{\circ} 1$}

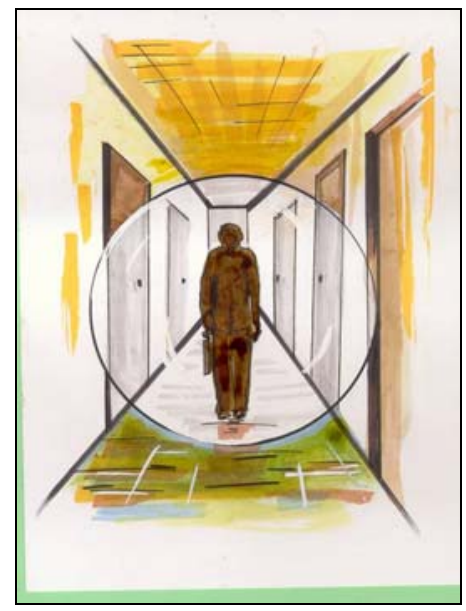

\section{Série $B-n^{\circ} 2$}

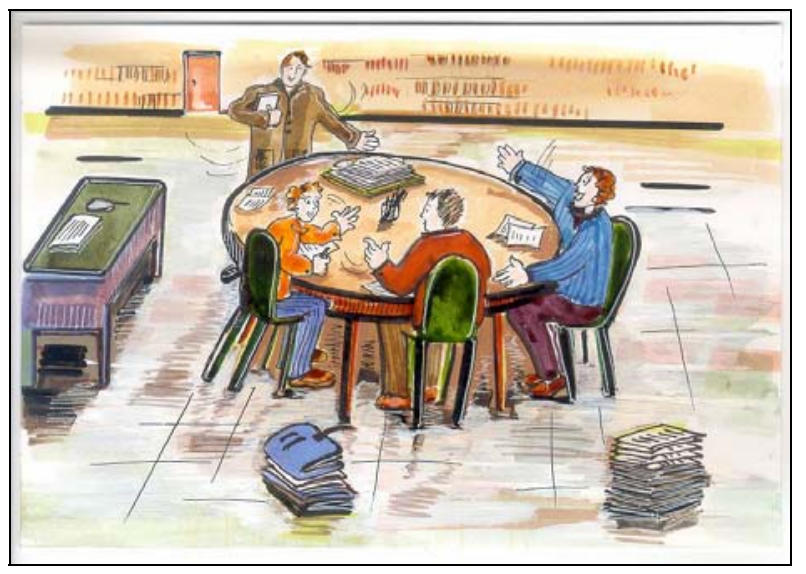

\section{Série B - n 3}

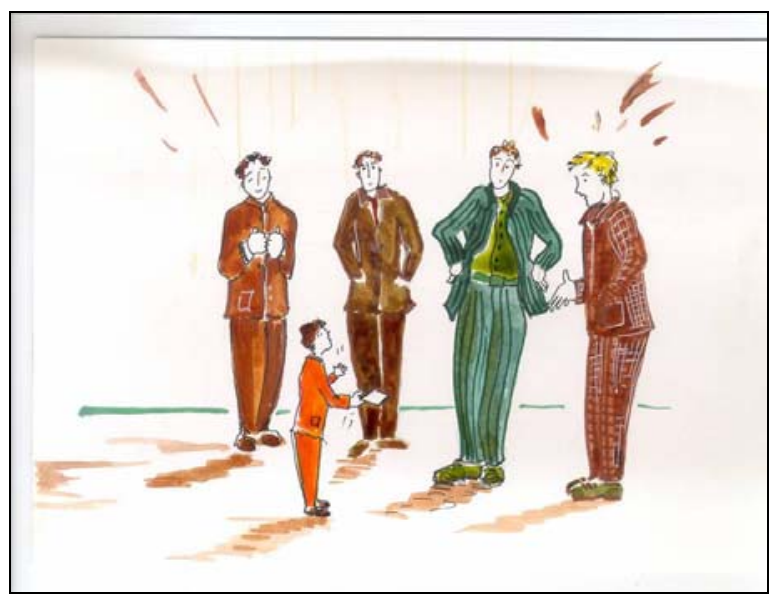




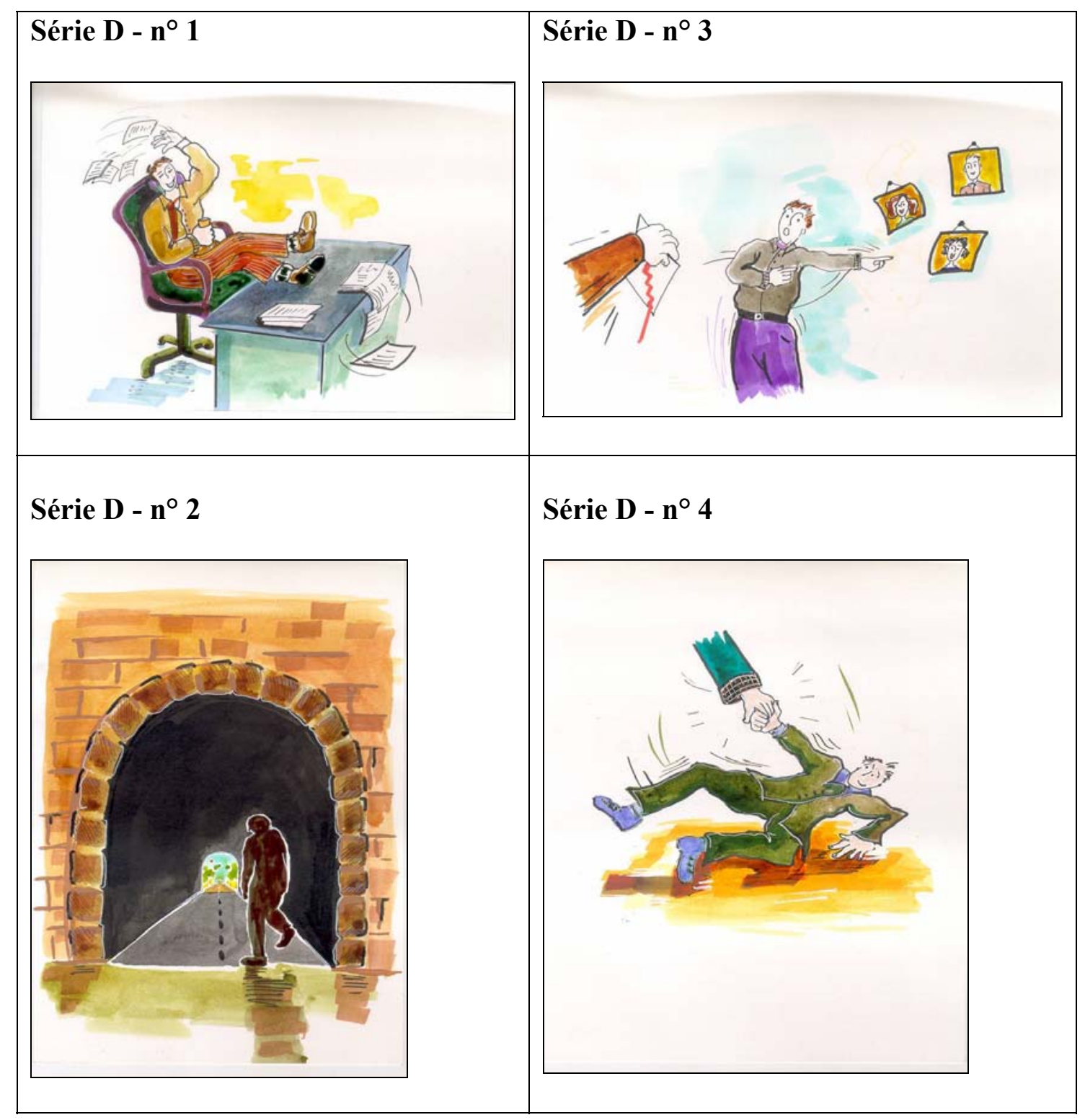




\section{BIBLIOGRAPHIE :}

Ahrens T., Dent J.F. (1998), « Accounting and Organizations: Realizing the Richness of Field Research », Vol 10, Journal of Management Accounting Research, pp. 1-39.

Anzieu et Chabert (1983), Les méthodes projectives, Presses Universitaires de France/ Le psychologue.

Aranya N. (1990), « Budget instrumentality, participation and organizational effectiveness », Journal of Management Accounting Research, 2, pp. 67-77.

Ardoin J.-L. (1995), «L'abus des centres de profit est dangereux pour la santé des entreprises », Revue Echanges, $\mathrm{n}^{\circ} 110$, mars, pp. 20-30.

Argyris C. (1952), The impact of budgets on people, Ithaca, New-York : The Controllership Foundation.

Berland N. (2001), « Les rôles du contrôle budgétaire : un modèle d'interprétation », Revue Française de Gestion, Septembre - Octobre, pp. $111-120$.

Berland N. (2002), Le contrôle budgétaire, Editions La Découverte.

Berland N., Pinçon J. (2002), « Gérer sans budget : l'exemple de Rhodia », Revue Echanges, ${ }^{\circ} 187$, avril, pp. $24-26$.

Bouquin H. (1999), « Théorie des organisations et contrôle », dans Encyclopédie de Comptabilité, Contrôle et Audit, sous la direction de Bernard Colasse.

Bouquin H. (2001), Le Contrôle de Gestion, $5^{\text {ème }}$ édition, Gestion PUF.

Briers M., Hirst M.K. (1990), « The role of budgetary information in performance evaluation », Accounting, Organizations and Society, 15, pp. 373-398.

Brownell P. (1982), « The role of accounting data in performance evaluation, budgetary participation and organizational effectiveness », Journal of Accounting Research, 20, pp. 12-27.

Brownell P. (1985), « Budgetary systems and the control of functionally differentiated organizational activities », Journal of Accounting Research, 23, pp. 502-512.

Brownell P., Dunk A.S. (1991), « Task uncertainty and its interactions with budgetary participation and budget emphasis : some methodological issues and empirical investigation », Accounting, Organizations and Society, 16, pp. 693-703.

Brownell P., Hirst M.K. (1986), « Reliance on Accounting Information, budgetary participation, and task uncertainty : tests of a three-way interaction », Journal of Accounting Research, 24, pp. 241-249.

Chapman C. S. (1997), « Reflexions on contingent view of accounting », Accounting, Organizations and Society, Vol. 22, n², pp. 189-205.

Dunbar R. L. M. (1971), « Budgeting for Control », Administrative Science Quarterly, March, pp. 88 - 96.

Dunk A.S. (1989), "Budget emphasis, budgetary participation and managerial performance: a note », Accounting, Organizations and Society, 14, pp. 321-324.

Dunk A.S. (1992), « Reliance on Budgetary Control, Manufacturing Process, Automation and Production Subunit Performance: a Research Note », Accounting, Organizations and Society, Vol. 17, n³/4, pp. 195 - 203.

Dunk A.S. (1993), « The effect of budget emphasis and information asymmetry on the relationship between budgetary participation and slack », The Accounting Review, 68, pp. 400-410.

Dupuy Y., Rolland G. (1991), Manuel de Contrôle de Gestion, Dunod.

Eisenhardt K.M. (1989), « Building theories from case study research », Academy of Management Review, Vol $14, \mathrm{n}^{\circ} 4$, pp. $532-550$.

Escoffier B. (1999) « Budgets et contrôle », dans Encyclopédie de Comptabilité, Contrôle et Audit, sous la direction de Bernard Colasse 
Fiol M., Lebas M. (1999), « Créer des situations de sens pour générer la performance : le double rôle du manager », pp. 70 - 76 dans Questions de contrôle, sous la Direction de Lionel Collins, PUF Gestion.

Gervais M. (1999), « Animation et gestion budgétaire », dans Encyclopédie de Comptabilité, Contrôle et Audit, sous la direction de Bernard Colasse

Gervais M. (2000), Contrôle de gestion, Economica, $7^{\mathrm{e}}$ édition, Paris.

Gignon-Marconnet I. (2003), « Les rôles actuels de la gestion budgétaire en France : une confrontation des perceptions de professionnels avec la littérature ", Comptabilité - Contrôle - Audit, Tome 9, Vol 1, pp. 53-78.

Giraud F., Saulpic O., Naulleau G., Delmond M.-H., Bescos P. (2002), Contrôle de gestion et Pilotage de la performance, Editions Gualino-Monstchrestien, Paris.

Govindarajan V. (1984), «Appropriateness of accounting data in performance evaluation: an empirical investigation of environmental uncertainty as an intervening variable », Accounting, Organizations and Society, 9, pp. 125-135.

Govindarajan V., Gupta A.K. (1985), «Linking control systems to business unit strategy: impact on performance », Accounting, Organizations and Society, 10, pp. 51-66.

Guelfant (1998), «Les études qualitatives marketing », Revue Française de Marketing, 168-169, pp. 51-60.

Guizani C., Brunhes-Faure M. (2002), « De la construction budgétaire au pilotage de la performance », Revue Echanges, ${ }^{\circ}$ 191, Août-Septembre, pp. 48-51.

Haire, M. (1950), « Projectives Techniques in Marketing Research », the Journal of Marketing, 14:5, april 1950

Hanson E. I. (1966), « The Budgetary Control Function », The Accounting Review, April, p p. 239-243.

Harrison G. L. (1992), « The cross-cultural generalizability of the relation between participation, budget emphasis and job-related attitudes », Accounting, Organizations and Society, 17, pp. 1-15.

Hartmann G.H. (2000), "The appropriateness of RAPM : toward the further development of theory », Accounting, Organizations and Society, 25, pp. 451-482.

Hayes R., Abernathy W. (1980), « Managing our way to economic decline », Harvard Business Review, Vol 58, $\mathrm{n}^{\circ} 4$, July-August, pp.67-77.

Hirst M.K. (1981), « Accounting information and the evaluation of subordinate performance: a situational approach », The Accounting Review, Vol 61, n, October, pp.771-784.

Hirst M. K. (1983), « Reliance on accounting performance measures, task uncertainty and dysfunctional behavior : some extensions », Journal of Accounting Research, 21, pp. 596-605.

Hirst M.K. (1987), « The effects of setting budget goals and task uncertainty on performance : a theoretical analysis », The Accounting Review, 62, pp. 774-784.

Hirst M.K., Yetton P. (1984), « Influence of reliance on accounting performance measures and job structure on role ambiguity for production and non-production jobs », Australian Journal of Management, 9, pp. 53-62.

Hofstede G. H. (1967), The Game of Budget Control, Assen, Van Gorcum.

Hope J., Fraser R. (2000), « Beyond Budgeting », Strategic Finance, October, pp. 30-35.

Hopwood A.G. (1972), «An empirical study of the role of accounting data in performance evaluation », Journal of Accounting Research, 10, pp. 156-182.

Imoisili O.A. (1989), « The role of budget data in the evaluation of managerial performance », Accounting, Organizations and Society, 14, pp. 325-335.

Iribarne (d') P. (1989), La logique de l'honneur : gestion des entreprises et traditions nationales, Paris, Le Seuil. Jordan H. (1998), Planification et contrôle de gestion en France en 1998, Cahier de recherche HEC, Jouy-enJosas, France. 
Kenis I. (1979), " Effects of budgetary goals characteristics on managerial attitudes and performance », The Accounting Review, 54, pp. 707-721.

Kren L. (1993), «Control system effects on budget slack », Advances in Management Accounting, Vol 2, pp. 109-118.

Langevin P., Naro G., "Contrôle et comportements : une revue de la littérature anglo-saxonne », Communication publiée dans les actes du 26 ème congrès de l'Association Française de Comptabilité, Louvain la Neuve, 22 et 23 mai 2003.

Lauzel P., Teller R. (1997), Contrôle de gestion et Budgets, Sirey.

Laverty K. J. (1996), «Economic short-termism: the debate, unresolved issues, and implications for management practice and research », Academy of Management Review, 21, pp. 825-860.

Lillis A.M., Mundy J. (2003), « Capitalising on the potential of cross-sectional field studies for theory refinement in management accounting ", Communication présentée au congrès de l'European Accounting Association, Séville, Avril 2003.

Lukka K. (1988), "Budgetary biasing in organizations: theoretical framework and empirical evidence », Accounting, Organizations and Society, Vol 13, n³, pp.281-301.

Malleret V. (1993), Une approche de la performance des services fonctionnels : l'évaluation des centres de coûts discrétionnaires, Thèse de Doctorat, Université Paris-Dauphine.

Merchant K. A. (1985), « Budgeting and the propensity to create budget slack", Accounting, Organizations and Society, 10, pp. 201-210.

Merchant K. A. (1990), « The effects of financial controls on data manipulation and management myopia », Accounting, Organizations and Society, 15, pp. 297-313.

Merchant K. A., Bruns W. J. (1986), « Measurements to cure management myopia », Business Horizons, MayJune, pp.56-64.

Merchant K.A., Manzoni J.-F. (1989), « The achievability of budget targets in profit centers: a field study », The Accounting Review, Vol 64, n³, July, pp. 539-558.

Milani K. (1975), « The Relationship of Participation in Budget-Setting to Industrial Supervisor Performance and Attitudes: a Field Study », The Accounting Review, April, pp. 274-284.

Naro G. (1998), « La dimension humaine du contrôle de gestion : la recherche anglo-saxonne sur les aspects comportementaux de la gestion budgétaire », Comptabilité-Contrôle-Audit, tome 4, volume 2, septembre 1998, pp. 45-69.

Onsi M. (1973), « Factor analysis of behavioral variables affecting budgetary slack », The Accounting Review, July, pp. 535-548.

Otley D. T. (1978), «Budget use and managerial performance », Journal of Accounting Research, 16, pp. $122-149$.

Otley D. T., Fakiolas A. (2000), «Reliance on accounting performance measures: dead end or new beginning ? ", Accounting, Organizations and Society, 25, pp. 497-510.

Ouchi W. (1980), «Markets, Bureaucracies and Clans », Administrative Science Quarterly, vol 25, n¹, pp. 129-141.

Ross A. (1995), « Job related tension, budget emphasis and uncertainty: a research note », Management Accounting Research, 6, pp. 1-11.

Segal J. P. (1987), « La gestion participative : une comparaison Etats-Unis, Québec, France », Revue Française de Gestion, $\mathrm{n}^{\circ} 64$, septembre - octobre, pp. 51-58.

Shields J. F., Shields M. D. (1998), « Antecedents of participative budgeting », Accounting, Organizations and Society, 23, pp. 49-76. 
Shields M.D., Young S.M. (1993), « Antecedents and consequences of participative Budgeting: Evidence on the Effects of Asymmetrical Information », Journal of Management Accounting Research, Fall, pp. 265-280.

Stedry A. C. (1960), Budget Control and Cost Behavior, Englewood Cliffs, NJ, Prentice-Hall.

Swieringa R. J., Moncur R. H. (1975), Some Effects of Participative Budgeting on Managerial Behavior, National Association of Accountants.

Thompson J.D. (1967), Organizations in action, McGraw-Hill, New-York.

Vagneur K., Peiperl H. (2000), « Reconsidering performance evaluative style », Accounting, Organizations and Society, 25, pp. 511-525.

Van Der Stede W.A. (2000), « The relationship between two consequences of budgetary controls : budgetary slack creation and managerial short-term orientation ", Accounting, Organizations and Society, 25, pp. 609-622.

Wallace M. E. (1966), « Behavioral Considerations in Budgeting », Management Accounting, August, pp. 3-8. 